\title{
Design of broadband high-gain GaN MMIC power amplifier based on reactive/resistive matching and feedback technique
}

\author{
Lin Peng ${ }^{1}$, Jianqiang Chen ${ }^{1}$, Zhihao Zhang ${ }^{1 a)}$, Yang Huang ${ }^{2}$, Tong Wang ${ }^{2}$, and Gary Zhang ${ }^{1}$
}

\begin{abstract}
This paper presents a K-band two-stage power amplifier (PA) with a compact circuit size of $1.8 \times 0.87 \mathrm{~mm}^{2}$. To guarantee broadband high-gain output performance, the optimal impedance domain and power cell are determined through load/source-pull simulation and $\mathrm{K}$ point method, respectively. Reactive/resistive matching networks are carefully employed to reduce the equivalent gate capacitance, improve stability and compensate for the device's negative gain roll-off slope. Meanwhile, combining with feedback technique adopted in driver stage, the entire operation bandwidth can be further extended. Under $12 \mathrm{~V}$ pulse voltage supply, $37.4 \%$ of peak power-added efficiency (PAE) at $26 \mathrm{GHz}$ and $24 \pm 0.5 \mathrm{~dB}$ of small-signal gain, 30.3-31.6 dBm of saturated output power $\left(\mathrm{P}_{\text {sat }}\right)$ across $22-27 \mathrm{GHz}$ are obtained as shown in the experimental results.

key words: gallium nitride $(\mathrm{GaN})$, monolithic microwave integrated circuit (MMIC), broadband power amplifier, feedback

Classification: Microwave and millimeter-wave devices, circuits, and hardware
\end{abstract}

\section{Introduction}

The extensive application and rapid development of wireless technology have led to the derivation of many types of communication systems. They usually adopt diverse standards or work in separated frequency ranges, resulting in mutual independence and thus requiring additional equipment for interconnection, which would increase costs and affect the operation reliability when close collaboration between distinct systems is necessary. In such context, broadband communication systems came into being and have been widely applied in modern commercial and military fields to date $[1,2,3]$. Besides, with the arrival of the $5 \mathrm{G}$ era, large bandwidth is also the essential ingredient to satisfy ever-growing mobile traffic volume demand [4, 5]. Therefore, broadband PAs are attracting more attention and gradually become the focus of research.

Due to the gain roll-off characteristics of transistor

\footnotetext{
${ }^{1}$ School of Information Engineering, Guangdong University of Technology, Guangzhou 510006, China

${ }^{2}$ Sichuan YiFeng Electronic Science \& Technology Co., LTD, Chengdu 610073, China

a) zhihaozhang@gdut.edu.cn
}

DOI: $10.1587 /$ elex.18.20210313

Received July 28, 2021

Accepted August 10, 2021

Publicized September 01, 2021 and its input and output impedances vary with frequency, how to achieve small-scale fully integrated 1-Watt PA with more than $20 \%$ fractional bandwidth and over 22 $\mathrm{dB}$ small-signal gain is the major issue to be solved in this study. Discussing the current mainstream of several typical broadband configurations, distributed amplifier has the widest bandwidth by incorporating the parasitic capacitances of multiple cells in parallel into artificial transmission lines, but it generally suffers from low gain, limited power level, and bad integration $[1,6,7,8]$. Although the balanced amplifier is capable of reaching one-octave bandwidth, it requires bulky $3 \mathrm{~dB}$ couplers and large power consumption $[5,9,10,11]$. The reactive/resistive topology can provide a positive roll-of insertion loss slope for gain compensation, but its modest bandwidth and relatively poor VSWR are the main drawbacks $[12,13]$. Another commonly used broadband amplification structure is negative feedback $[2,14,15$, 16], which offers obvious advantages in terms of cost, space, and complexity. However, the introduction of a feedback resistor brings the problem of gain degradation that needs to be tackled by the active device with large transconductance. In this regard, GaN, the thirdgeneration wide-bandgap semiconductor technology, with superior breakdown voltage and electron mobility properties is the promising candidate. Moreover, GaNbased devices also have the inherent benefit of high output resistance along with low parasitic capacitance, which makes them ideal for broadband amplifier design [1, 17, 18]. Accordingly, we propose a solution that combines reactive/resistive gain equalizing networks in the form of parallel RC and negative feedback technique to realize a cascaded two-stage MMIC PA covering 22$27 \mathrm{GHz}$ based on $0.1 \mu \mathrm{m}$ GaN high electron mobility transistor (HEMT) process on a $100-\mu \mathrm{m}$-thick silicon substrate developed by OMMIC.

\section{Analysis and design of broadband PA}

\subsection{Unit cell selection}

Concerning the requirements of miniaturization and good PAE, it's preferable to adopt two-stage common-source architecture for PA. In doing so, there may be a risk of 
insufficient gain owing to the inevitable loss of matching networks (MNs) and the maximum available gain (MAG) of a single transistor. In consequence, the total gate width (TGW) of power stage cell should be selected as small as possible under the premise of meeting $1 \mathrm{~W}$ $\mathrm{P}_{\text {sat }}$ and is calculated to be $370 \mu \mathrm{m}$ when given $1 \mathrm{dBm}$ trade-off margin with a moderate power density of 3.4 $\mathrm{W} / \mathrm{mm}$. Higher optimal impedance can be obtained concurrently, which helps simplify matching. Note, TGW is the product of unit gate width (UGW) and the number of gate fingers (NGF), which impacts the device's gain transition from maximum stable gain (MSG) into MAG. Since the turning frequency point involved is exactly where the Rollett's stability factor $\mathrm{K}$ becomes unity, we call it K-point, which is better to fall outside the upper band boundary to ensure that the active device has enough intrinsic gain [19]. The simulated dependence of the K-point on TGW of three kinds of unit cells is depicted in Fig. 1, from which we can conclude that using devices with larger TGW is less suitable for higher frequencies due to the falling K-point.

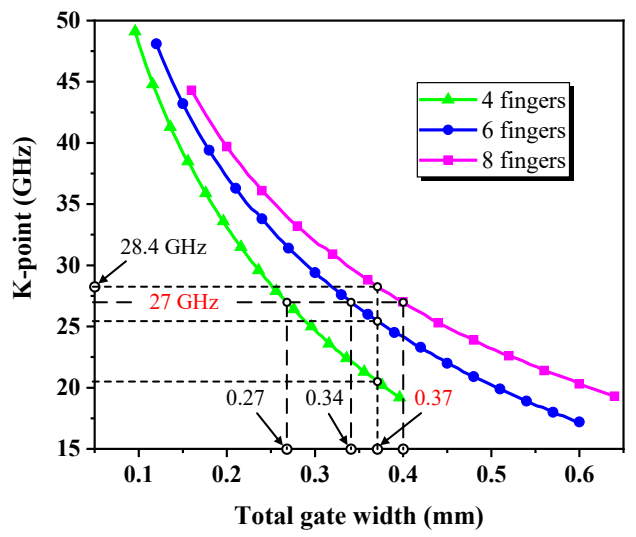

Fig. 1. Simulation of the dependence of the K-point on TGW.

On the other hand, when the gate periphery is set, as the NGF increases by a multiple of 2 for the sake of layout symmetry, there is an increasing phase difference between gate fingers and the middle sources of transistor have a long electrical path to via-hole ground, extra parasitic inductance will be generated, leading to a reduction in forward gain. Yet at the same time, the decrease of UGW helps to avoid excessive phase errors as well as signal propagation fade along the gate, then the most amount of gain can be preserved [20]. Taking into account these factors, Fig. 1 reveals that minor UGW is the dominant contributor to a higher K-point for the same TGW. According to device scaling rules of $\mathrm{UGW} \leq 100 \mu \mathrm{m}$ and $\mathrm{NGF} \leq 8$ recommended by the foundry design manual in conjunction with TGW of 370 $\mu \mathrm{m}$, the corresponding three transistors and their K-point information are summarized in Table I. Among them, the 8 -finger FET cell was chosen for output stage, a $46 \times 4$ $\mu \mathrm{m}$ cell was thereafter assigned in prior stage to deliver adequate linear power depending on a conservative staging ratio of $1: 2$ on account of soft compression phenomena of GaN-based devices. It is worth mentioning that each cell was biased at equal $-1 \mathrm{~V} \mathrm{~V}_{\mathrm{gs}}$ to yield maximum transconductance and thus elevated power gain as suggested in Fig. 2.

Table I. K-points corresponding to different fingers of unit cells with equal TGW.

\begin{tabular}{c|c|c}
\hline NGF & UGW (um) & K-point $(\mathrm{GHz})$ \\
\hline 4 & 92 & 20.7 \\
\hline 6 & 62 & 25.4 \\
\hline 8 & 46 & 28.4 \\
\hline
\end{tabular}

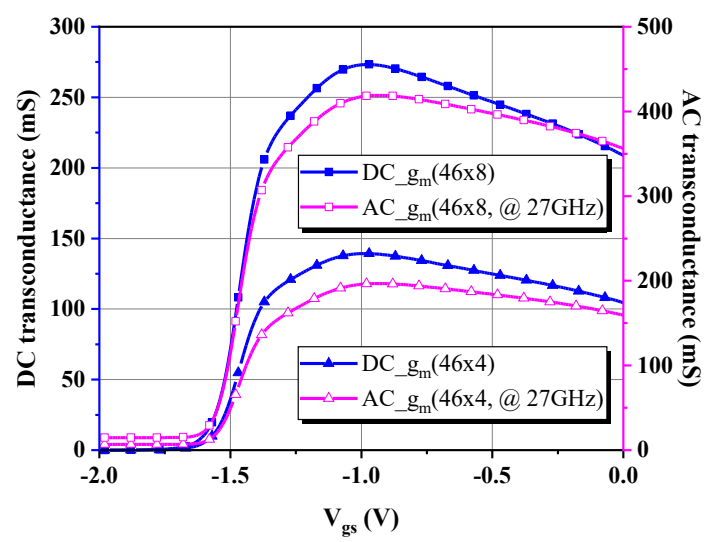

Fig. 2. Simulated DC/AC transconductance $g_{m}$ for a $46 \times 8 \mu \mathrm{m}$ HEMT at $\mathrm{V}_{\mathrm{ds}}=12 \mathrm{~V}$.

\subsection{Parallel RC gain equalizing network design}

To flatten the gain response inside the specified bandwidth, we employed a parallel RC gain equalizing network placed in the input terminal of transistor, which also has the function of improving stability and reducing parasitic effects, primarily the gate-source capacitance $\mathrm{C}_{\mathrm{gs}}$. To strike a balance between these three aspects while maintaining reasonable MAG, the simplified smallsignal equivalent circuit of a GaN HEMT is first given in Fig. 3 including seven intrinsic frequency-dependent elements which can be analytically extracted through Yparameters obtained from S-parameter simulation of nonlinear FET model for a certain bias point [21].

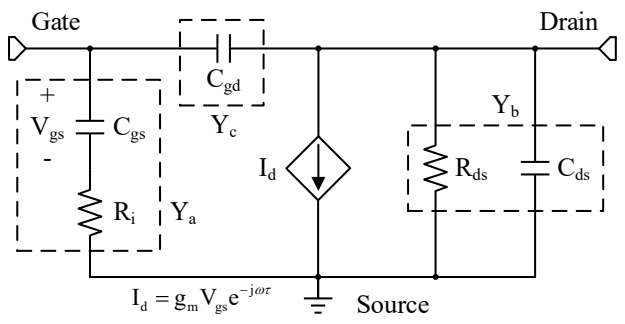

Fig. 3. Equivalent circuit model of a GaN HEMT.

$$
[\mathrm{Y}]_{\mathrm{HEMT}}=\left[\begin{array}{ll}
y_{11} & y_{12} \\
y_{21} & y_{22}
\end{array}\right]=\left[\begin{array}{cc}
Y_{a}+Y_{c} & -Y_{c} \\
g_{m}-Y_{c} & Y_{b}+Y_{c}
\end{array}\right]
$$


Based on Fig. 3 and Eq. (1), channel resistance $\mathrm{R}_{\mathrm{i}}$, depletion capacitance $\mathrm{C}_{\mathrm{gs}}$ and $\mathrm{C}_{\mathrm{gd}}$ can be expressed as

$$
\begin{gathered}
\mathrm{R}_{\mathrm{i}}=\operatorname{Re}\left(\frac{1}{y_{11}+y_{12}}\right) \\
\mathrm{C}_{\mathrm{gs}}=\left[-\omega \operatorname{Im}\left(\frac{1}{y_{11}+y_{12}}\right)\right]^{-1} \\
\mathrm{C}_{\mathrm{gd}}=\frac{1}{\omega} \operatorname{Im}\left(-y_{12}\right)
\end{gathered}
$$

Using Eq. (2) to (4), $\mathrm{R}_{\mathrm{i}}=5.3 \Omega, \mathrm{C}_{\mathrm{gs}}=0.655 \mathrm{pF}$, and $\mathrm{C}_{\mathrm{gd}}=$ $0.087 \mathrm{pF}$ for a $46 \times 8 \mu \mathrm{m}$ HEMT are derived at $27 \mathrm{GHz}$ under $12 \mathrm{~V}$ supply. Because $\mathrm{C}_{\mathrm{gd}}$ is rather smaller than $\mathrm{C}_{\mathrm{gs}}$, the cell is treated as a unilateral two-port network for simplicity, and the input impedance $Z_{\text {in }}$ seen from the gate node of transistor is therefore modeled by means of a series connection of $R_{i}$ and $C_{g s}$.

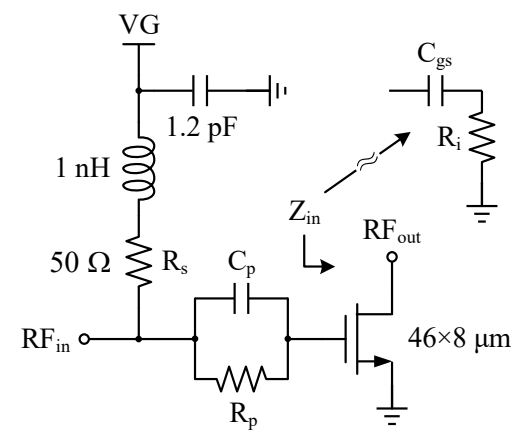

Fig. 4. Schematic of the output stage cell with parallel-RC and seriesRL stabilization networks.

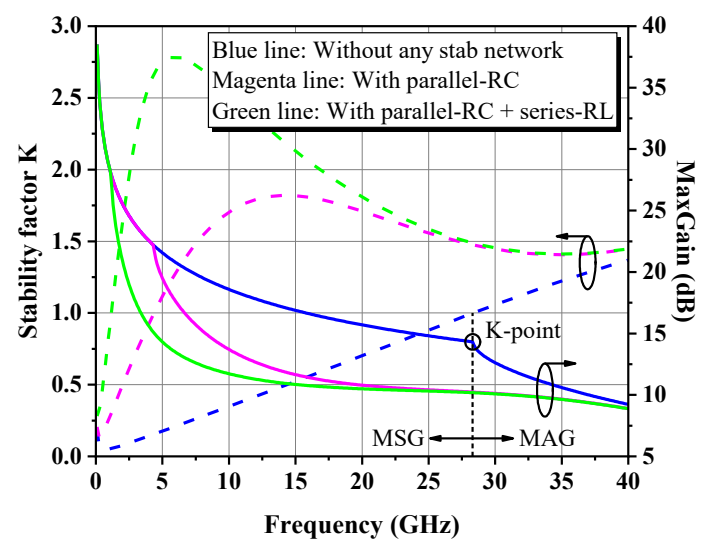

Fig. 5. Simulated stability factor $K$ and maximum gain of the $46 \times 8 \mu \mathrm{m}$ $\mathrm{GaN}$ transistor before and after the insertion of stabilization networks.

In order to lower the equivalent gate capacitance so as to ease interstage matching, the value of $C_{p}$ in parallel RC network presented in Fig. 4 should be less than $\mathrm{C}_{\mathrm{gs}}$, while the thin-film resistor $R_{p}$ is responsible to absorb low-frequency gain and reduce negative resistance that appears at device gate [1]. Nevertheless, $C_{p}$ could not be too small, otherwise the wanted signal will be greatly attenuated by $R_{p}$. For this reason, putting identical emphasis on bypassing the in-band RF component is necessary, thereby we take MAG $>10 \mathrm{~dB}$ at the upper edge of the operation band as another constraint for $C_{p}$ selection and with the adjustment of $\mathrm{R}_{\mathrm{p}}$ together to facilitate uniform gain at the expense of acceptable loss. Finally, $C_{p}$ and $R_{p}$ were respectively determined to be $0.47 \mathrm{pF}$ and $25 \Omega$ in a compromise. The gate bias branch formed by $50 \Omega$ resistor $R_{s}$ and choke spiral inductor in series mainly aims to further eliminate potential spurious oscillations and prevent the leakage of RF energy [22, 23], whereas it has negligible effect on the amplification chain within the band of interest. Fig. 5 plots detailed comparisons of the foresaid stabilization measures.

\subsection{Analysis and design of the feedback loop}

A lossy feedback network from the drain to the gate of the device is another effective way in controlling gain ripple, amplifier stability, and matching which also contributes to making the circuit insensitive to ambient temperature and process variation [24, 25], bringing a significant improvement in robustness. So a feedback loop composed of L-R-C in series is introduced connected to the $46 \times 4 \mu \mathrm{m}$ cell, as illustrated in Fig. 6 .

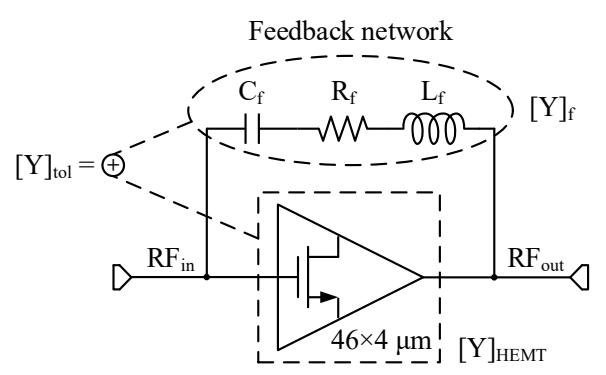

Fig. 6. Simplified schematic diagram of the driver stage cell with a shunt feedback network based on L-R-C in series.

The total admittance of the transistor with feedback is the sum of the individual admittances of the open-loop amplifier and shunt feedback network, written as

$$
\begin{aligned}
{[\mathrm{Y}]_{\mathrm{tol}} } & =\left[\begin{array}{ll}
y_{t 11} & y_{t 12} \\
y_{t 21} & y_{t 22}
\end{array}\right]=[\mathrm{Y}]_{\text {HEMT }}+[\mathrm{Y}]_{\mathrm{f}} \\
& =\left[\begin{array}{ll}
y_{11}+y_{f} & y_{12}-y_{f} \\
y_{21}-y_{f} & y_{22}+y_{f}
\end{array}\right]
\end{aligned}
$$

where $\mathrm{y}_{\mathrm{f}}$ is the feedback admittance given by

$$
y_{f}=\left(R_{f}+j \omega L_{f}+\frac{1}{j \omega C_{f}}\right)^{-1}
$$

Thus, the stability factor $\mathrm{K}$ and $\mathrm{S}_{21}$ of this parallel circuit are described as follows $[2,26]$

$$
\begin{gathered}
K=\frac{2 \operatorname{Re}\left(y_{t 11}\right) \operatorname{Re}\left(y_{t 22}\right)-\operatorname{Re}\left(y_{t 12} y_{t 21}\right)}{\left|y_{12} y_{21}\right|} \\
S_{21}=\frac{-2 Y_{0} y_{t 21}}{\left(Y_{0}+y_{t 11}\right)\left(Y_{0}+y_{t 22}\right)-y_{t 12} y_{t 21}}
\end{gathered}
$$


Recall the expression of transducer gain, we have [12]

$$
G_{T}=\frac{\left|S_{21}\right|^{2}\left(1-\left|\Gamma_{S}\right|^{2}\right)\left(1-\left|\Gamma_{L}\right|^{2}\right)}{\left|\left(1-S_{11} \Gamma_{S}\right)\left(1-S_{22} \Gamma_{L}\right)-S_{12} S_{21} \Gamma_{S} \Gamma_{L}\right|^{2}}
$$

If both the source and load are matched to characteristic impedance $Z_{0}$, the reflection coefficient $\Gamma_{\mathrm{S}}=\Gamma_{\mathrm{L}}=0$ and $\mathrm{G}_{\mathrm{T}}$ simply becomes $\left|S_{21}\right|^{2}$. From Eq. (5) to (9), the desired gain response and stability enhancement can be achieved by properly tuning the feedback components. In design, $0.5 \mathrm{nH}$ inductor was added for the purpose of reducing the effectiveness of negative feedback with increasing frequency, while the $0.41 \mathrm{pF}$ capacitor not only serves as a DC block to keep the isolation between the gate and drain bias but also regulates the phase and magnitude of feedback waveform. Next, $57 \Omega$ resistor was selected to raise the valley $\mathrm{K}$-factor within $10-30 \mathrm{GHz}$ to slightly below 1 without sacrificing too much gain and smooth the frequency response. In addition, care must be taken to check that there are no frequencies where the feedback could cause a regenerative unstable condition.

\subsection{Design strategy for matching networks}

The output capability of PA depends to a large extent on output $\mathrm{MN}$, we started by determining the optimal complex impedance domain in the range of $22-28 \mathrm{GHz}$ to ensure that PA's performance at $27 \mathrm{GHz}$ still fully meets the specifications even in the worst case. Iterative load/source-pull procedures of the stabilized GaN HEMT with an input power about $\mathrm{P}_{1 \mathrm{~dB}}$ were carried out at low, medium, and high fundamental frequency points, whereas the influence of harmonics was ignored as they locate away from the passband and can be easily filtered out $[3,27,28]$.

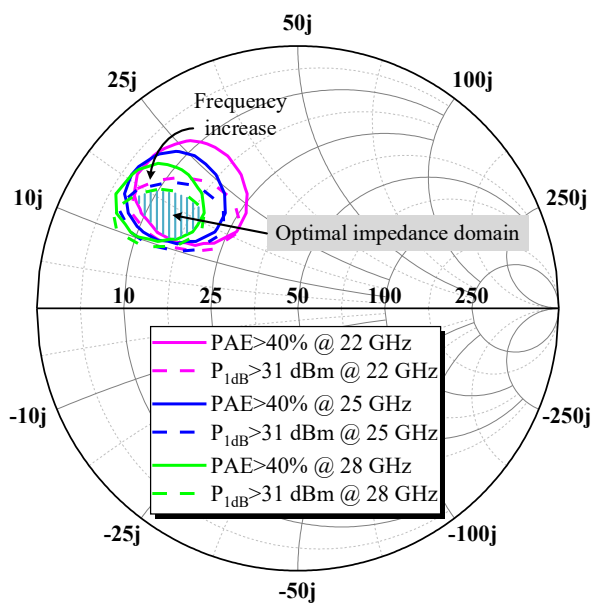

Fig. 7. Optimal load impedance of fundamental.

From Fig. 7, it is clearly observed that the contour for load impedances whose inside fulfill the set goal of either $\mathrm{P}_{1 \mathrm{~dB}}>31 \mathrm{dBm}$ or $\mathrm{PAE}>40 \%$ is constantly getting narrower and moving in a counter-clockwise direction towards the real axis of the Smith Chart attributed to the strengthening innate output parasitics as frequency increases, which explains the tolerance region defined by the overlapping portion of three groups of contours is mostly restricted by the contours at $28 \mathrm{GHz}$. That means broadband matching tends to match at high-frequency side, wider bandwidth can be produced through appropriately modifying the degree of mismatch at low frequencies. Subsequently, $Z_{\mathrm{L}, \mathrm{opt}}=12+\mathrm{j} * 14.6 \Omega$ and $\mathrm{Z}_{\mathrm{S}, \mathrm{opt}}=4.5+\mathrm{j} * 11.2 \Omega$ were selected as the initial targeted center values. The output $\mathrm{MN}$ transforms $50 \Omega$ to $\mathrm{Z}_{\mathrm{L}, \mathrm{opt}}$ with series microstrip lines and shunt low capacitance density capacitors instead of open-circuited stubs for shrinking the occupation area, and plays a vital role in suppressing harmonic distortion, while the interstage MN was synthesized to conjugately match two devices for maximum gain. As regards input $\mathrm{MN}$, is intended to improve the terminal return loss. After cascading all independently designed MNs with two transistors in order, the stagger-tuning technique was undertaken to realize the mutual compensation of the insertion loss of MNs in different frequency ranges with the consideration of microstrip discontinuities, cross-coupling, and actual wedge-bonding wires, broadband gain response can be achieved eventually. In particular, the drain feed traces used double-layer metal with a total thickness of $2.5 \mu \mathrm{m}$ to handle high current swing. At last, we conducted a thorough nonlinear EM co-simulation of the complete layout shown in Fig. 8 to verify the amplifier was unconditionally stable under any possible levels of incident power throughout the whole band.

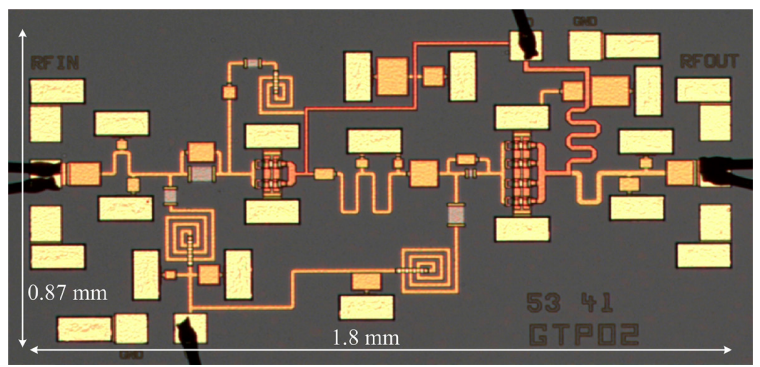

Fig. 8. Micrograph of the proposed PA (dimensions: $1.8 \times 0.87 \mathrm{~mm}^{2}$ ).

\section{Fabrication and measurement results}

Fig. 9 is the photo of a bare-die chip mounted to the test fixture. Gate bias voltage was adjusted to reach the same quiescent current draw of $170 \mathrm{~mA}$ as in simulation under $12 \mathrm{~V}$ supply in pulse mode with $5 \%$ duty-cycle and $1 \mathrm{~ms}$ duration to avoid overheating during continuous tests. Small-signal results in Fig. 10 exhibit that the fabricated PA features $24 \pm 0.5 \mathrm{~dB}$ linear gain over $22-27 \mathrm{GHz}$, and the input and output reflection coefficients are less than $9.2 \mathrm{~dB}$ and $-11.3 \mathrm{~dB}$, respectively. Compared with the simulated one, measured $\mathrm{S}_{21}$ has a low-frequency offset of approximately $0.8 \mathrm{GHz}$ and is overall higher, but the trends of the two curves are basically consistent. 


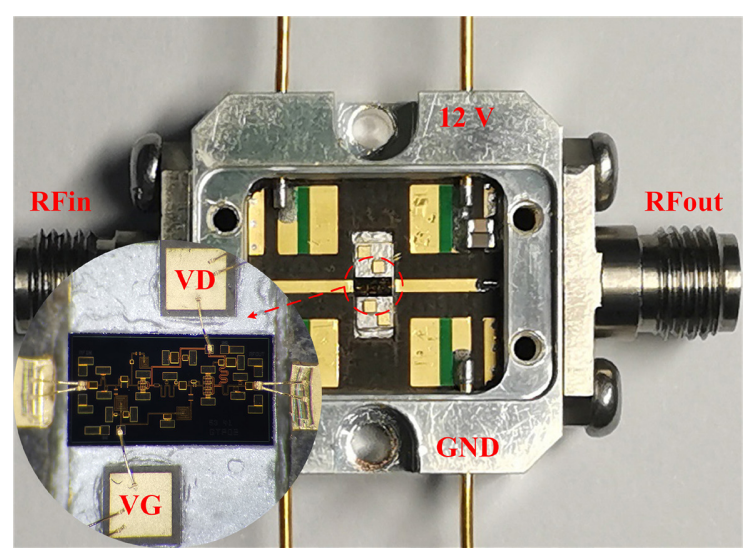

Fig. 9. Implemented MMIC PA inside the test fixture.

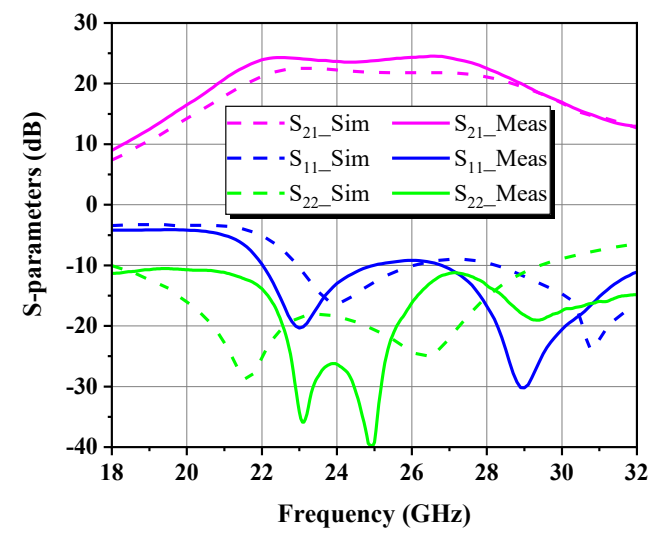

Fig. 10. Simulation and measurement results of S-parameter.

In the same band and at room temperature, Fig. 11 and Fig. 12 indicate that $\mathrm{P}_{4 \mathrm{~dB}}$ exceeds $28.3 \mathrm{dBm}, \mathrm{P}_{\text {sat }}$ fluctuates between $30.3-31.6 \mathrm{dBm}$, the PAE at $\mathrm{P}_{\text {sat }}$ reaches $30.5 \%-36.9 \%$. Notably, the best performance of designed PA occurs at $26 \mathrm{GHz}$, corresponding power sweep characteristic curve is plotted in Fig. 13, where the peak PAE approaches $37.4 \%$ found around $\mathrm{P}_{6 \mathrm{~dB}}$ at an input power of $14 \mathrm{dBm}$. Table II lists the performance comparison of some related PAs.

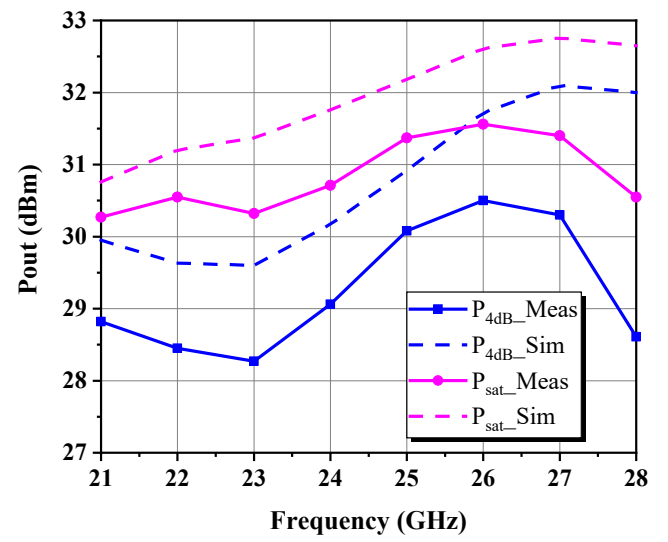

Fig. 11. Measured and simulated $\mathrm{P}_{\text {out }}$ at $4 \mathrm{~dB}$ gain compression and saturation state.

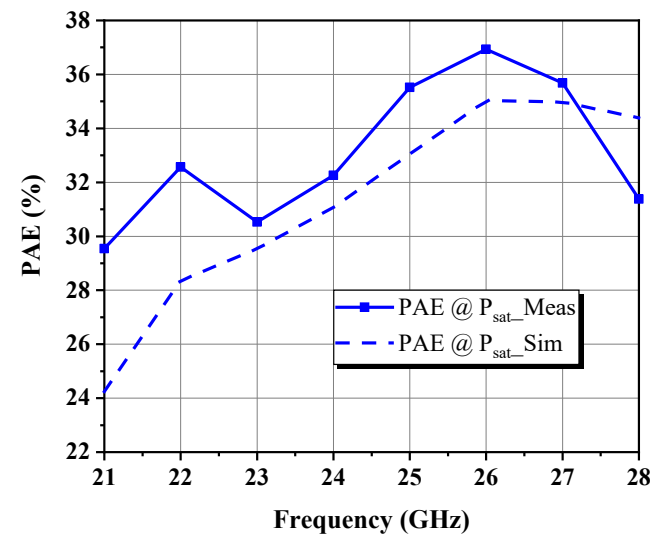

Fig. 12. Measured and simulated PAE at $P_{\text {sat }}$ as a function of frequency.

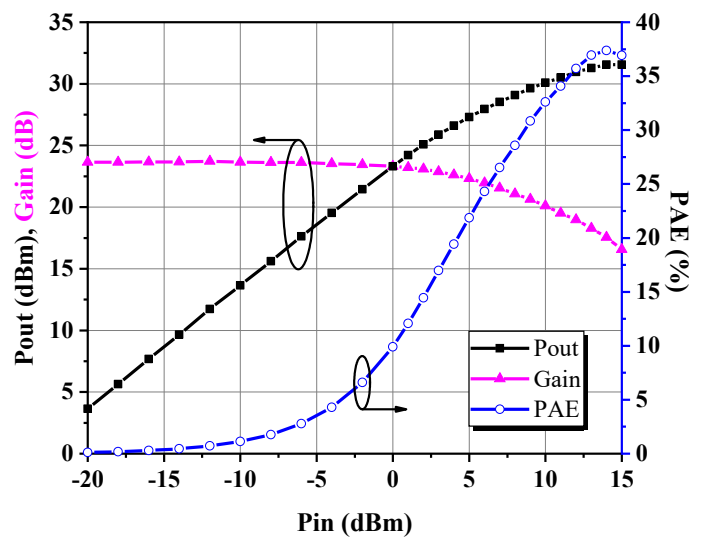

Fig. 13. Measured $\mathrm{P}_{\text {out }}$, PAE, and gain behaviors with respect to input power at $26 \mathrm{GHz}$.

Table II. Comparison with previous studies.

\begin{tabular}{c|c|c|c|c|c}
\hline Ref. & $\begin{array}{c}\text { Freq. } \\
(\mathrm{GHz})\end{array}$ & $\begin{array}{c}\text { FBW } \\
(\%)\end{array}$ & $\begin{array}{c}\text { Gain } \\
(\mathrm{dB})\end{array}$ & $\begin{array}{c}\mathrm{P}_{\text {out }} \\
(\mathrm{dBm})\end{array}$ & $\begin{array}{c}\text { PAE } \\
(\%)\end{array}$ \\
\hline$[9]$ & $32-38$ & 17.1 & $17 \pm 0.5$ & $36.2-37.2$ & $25-34$ \\
\hline$[17]$ & $29-34$ & 15.9 & $27 \pm 1$ & $37-39.5$ & $30-35.5$ \\
\hline$[29]$ & $25-29$ & 14.8 & $22.7 \pm 0.7$ & $25.6-26.3$ & $27.2-32.5$ \\
\hline$[30]$ & $19-23$ & 19.1 & $23.6 \pm 1.4$ & $35.1-37.3$ & $20-29.8$ \\
\hline This work & $22-27$ & 20.4 & $24 \pm 0.5$ & $30.3-31.6$ & $30.5-36.9$ \\
\hline
\end{tabular}

\section{Conclusion}

A two-stage broadband PA operating in $22-27 \mathrm{GHz}$ band is demonstrated and fabricated in a $0.1 \mu \mathrm{m}$ GaN HEMT process. To obtain high gain, the optimum device geometry is evaluated based on K-point method to derive a perfect combination of NGF and UGW after specifying the right TGW. Parallel RC gain equalizing networks and feedback technique are both utilized to get gain flatness and enhance stability simultaneously. The concept of optimal impedance domain is applied in the design to achieve broadband output performance. With the help of multistage matching networks, the transistor's gain rolloff characteristics can be further compensated while accomplishing low-Q impedance transformation. The $1.57 \mathrm{~mm}^{2}$ MMIC chip exhibits a measured small-signal gain of $24 \mathrm{~dB}$ averagely with $0.5 \mathrm{~dB}$ variation across 22 
$27 \mathrm{GHz}$ and delivers more than $30.3 \mathrm{dBm} \mathrm{P}_{\text {sat }}$ associated with $30.5 \%-36.9 \%$ of PAE which match the simulation expectations with a nice agreement. Measurement results prove that the proposed compact broadband PA fits the practical K-band transmitter systems.

\section{Acknowledgments}

This work was funded by the Key-Area Research and Development Program of Guangdong Province (No. 2018B010115001) and the National Key R\&D Program of China (2018YFB1802100). The authors would like to thank the staff of Sichuan YiFeng Electronic Science \& Technology Co., LTD for their kind assistance and support to this work.

\section{References}

[1] D.-H. Shin, et al.: "6-GHz-to-18-GHz AlGaN/GaN cascaded nonuniform distributed power amplifier MMIC using load modulation of increased series gate capacitance," ETRI J. 39 (2017) 737 (DOI: 10.4218/etrij.17.0116.0737).

[2] K. Kim, et al.: "Design of a broadband power amplifier using an optimized feedback network," Microw. Opt. Technol. Lett. 53 (2011) 2846 (DOI: 10.1002/mop.26372).

[3] X. Ding, et al.: " $2-4 \mathrm{GHz}$ wideband power amplifier with ultraflat gain and high PAE," Electron. Lett. 49 (2013) 326 (DOI: 10.1049/el.2012.4135).

[4] M. Shafi, et al:: "5G: A tutorial overview of standards, trials, challenges, deployment, and practice," IEEE J. Sel. Areas Commun. 35 (2017) 1201 (DOI: 10.1109/JSAC.2017.2692307).

[5] A. Alizadeh, et al.: "On design of wideband compact-size Ka/Qband high-power amplifiers," IEEE Trans. Microw. Theory Techn. 64 (2016) 1831 (DOI: 10.1109/TMTT.2016.2554578).

[6] K.-T. Bae and D.-W. Kim: "2-6 GHz GaN distributed power amplifier MMIC with tapered gate-series/drain-shunt capacitors," Int. J. RF Microw. Comput.-Aided Eng. 26 (2016) 456 (DOI: 10.1002/mmce.20989).

[7] Y. Chen, et al.: "A broadband GaAs high power millimeter wave amplifier with high gain and flatness," IEICE Electron. Express 15 (2018) 20180229 (DOI: 10.1587/elex.15.20180229).

[8] C. F. Campbell: "Evolution of the nonuniform distributed power amplifier: a distinguished microwave lecture," IEEE Microw. Mag. 20 (2019) 18 (DOI: 10.1109/MMM.2018.2875619).

[9] S. Chen, et al.: "High efficiency $5 \mathrm{~W} / 10 \mathrm{~W} 32-38 \mathrm{GHz}$ power amplifier MMICs utilizing advanced $0.15 \mu \mathrm{m}$ GaN HEMT technology," Proc. IEEE CSICS Conf. (2016) 1 (DOI: 10.1109/CSICS.2016.7751040).

[10] P. H. Pednekar, et al.: "RF-input load modulated balanced amplifier with octave bandwidth," IEEE Trans. Microw. Theory Techn. 65 (2017) 5181 (DOI: 10.1109/TMTT.2017.2748123).

[11] G. Wang and H. Tao: "An X-band high-efficiency GaN load modulated balanced amplifier MMIC," Microw. Opt. Technol. Lett. 62 (2020) 2195 (DOI: 10.1002/mop.32309).

[12] I. J. Bahl: Fundamentals of $R F$ and Microwave Transistor Amplifiers (Wiley, Hoboken, NJ, USA, 2009) 37, 298.

[13] Z. Cheng, et al.: "A 3-7 GHz GaN HEMT power amplifier," Microw. Opt. Technol. Lett. 58 (2016) 901 (DOI: 10.1002/mop.29699).

[14] K. B. Niclas, et al.: "The matched feedback amplifier: Ultrawideband microwave amplification with GaAs MESFET's," IEEE Trans. Microw. Theory Techn. 28 (1980) 285 (DOI: 10.1109/TMTT.1980.1130067).
[15] Y. J. Qiu, et al.: "Compact hybrid broadband GaN HEMT power amplifier based on feedback technique," Electron. Lett. 49 (2013) 372 (DOI: 10.1049/el.2012.3910).

[16] J. Hu, et al.: "Analysis and design of a $0.1-23 \mathrm{GHz}$ LNA MMIC using frequency-dependent feedback," IEEE Trans. Circuits Syst. II, Exp. Briefs $66 \quad$ (2019) $1517 \quad$ (DOI: 10.1109/TCSII.2019.2891262).

[17] N. Estella, et al.: "High-efficiency, Ka-band $\mathrm{GaN}$ power amplifiers," Proc. IEEE MTT-S Int. Microw. Symp. (2019) 568 (DOI: 10.1109/MWSYM.2019.8701005).

[18] K. H. Hamza and D. Nirmal: "A review of GaN HEMT broadband power amplifiers," AEU - Int. J. Electron. Commun. 116 (2020) 153040 (DOI: 10.1016/j.aeue.2019.153040).

[19] P. Dennler: "Analysis of the potential of gallium nitride based monolithic power amplifiers in the microwave domain with more than an octave bandwidth," Ph.D Dissertation, University of Freiburg, Freiburg (2014).

[20] S. P. Marsh: "MMIC power splitting and combining techniques," Proc. IEE Tutorial Colloq. Design RFIC's MMIC's (1997) 6/1 (DOI: 10.1049/ic:19971364).

[21] K.-W. Yeom: Microwave Circuit Design: A Practical Approach Using ADS (Prentice-Hall, Englewood Cliffs, NJ, USA, 2015) 1st ed. 260.

[22] G. Nikandish and A. Medi: "A design procedure for highefficiency and compact-size 5-10-W MMIC power amplifiers in GaAs pHEMT technology," IEEE Trans. Microw. Theory Techn. 61 (2013) 2922 (DOI: 10.1109/TMTT.2013.2271997).

[23] A. Yousefi and A. Medi: "Wide-band high-efficiency ku-band power amplifier," IET Circuits Devices Syst. 8 (2014) 583 (DOI: 10.1049/iet-cds.2014.0134).

[24] C. W. Huang, et al.: "A Ku-band four-stage temperature compensated PHEMT MMIC power amplifier," Microw. Opt. Technol. Lett. 44 (2005) 480 (DOI: 10.1002/mop.20673).

[25] M. L. Bhavsar, et al.: "A Ku-band 1-Watt two stage power amplifier MMIC for space with 52\% Power added efficiency," Asia-Pacific Microw. Conf. (2016) 1 (DOI: 10.1109/APMC.2016.7931325).

[26] H. F. Hammad, et al.: "Feedback for multiband stabilization of CS and CG MESFET transistors," IEEE Microw. Wireless Compon. Lett. 12 (2002) 122 (DOI: 10.1109/7260.993288).

[27] M. Zhang and X. Huang: "Design of broadband high-efficiency power amplifier based on elliptic low-pass and band-pass matching network," IEICE Electron. Express 16 (2019) 20190455 (DOI: 10.1587/elex.16.20190455).

[28] J. Xia, et al.: "A linearized 2-3.5 GHz highly efficient harmonictuned power amplifier exploiting stepped-impedance filtering matching network," IEEE Microw. Wireless Compon. Lett. 24 (2014) 602 (DOI: 10.1109/LMWC.2014.2324752).

[29] L. Peng, et al.: "Ka-band GaAs MMIC driver amplifier design," IEEE Int. Conf. Electron. Inf. Commun. Technol. (2020) 287 (DOI: 10.1109/ICEICT51264.2020.9334274).

[30] Z. Huang, et al.: "A 4 W K-band GaAs MMIC power amplifier with 22 dB gain," J. Semicond. 31 (2010) 035001-1 (DOI: 10.1088/1674-4926/31/3/035001). 